International Journal of Pure and Applied Mathematics

Volume 85 No. 4 2013, 669-678

ISSN: 1311-8080 (printed version); ISSN: 1314-3395 (on-line version)

url: http://www.ijpam.eu

doi: http://dx.doi.org/10.12732/ijpam.v85i4.4

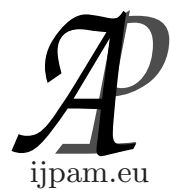

\title{
A SOLVABLE QSDE THROUGH SEMIGROUPS OF OPERATORS AND SOME PHYSICAL APPLICATIONS
}

\author{
O. González-Gaxiola ${ }^{1}$, José A. Santiago ${ }^{2}$, G. Chacón-Acosta ${ }^{3}$ \\ ${ }^{1,2,3}$ Department of Applied Mathematics and Systems \\ UAM-Cuajimalpa \\ Artificios 40, México, D.F. 01120, MEXICO
}

\begin{abstract}
In this paper we give a characterization, through semigroups theory, of the solution of a quantum stochastic differential equation (QSDE). In the physical interpretation of the problem, we show that the group that characterizes the quantum dynamics appears as the strong limit a family of translations perturbed by a Gaussian potential. Finally, we use the model to study a twolevel atom in an electromagnetic field.
\end{abstract}

AMS Subject Classification: 81Q10, 81Q80, 81V80

Key Words: quantum stochastic differential equation, number process, evolution equation, exponential vector

\section{Introduction}

The QSDE, like the Hudson-Parthasarathy equation, has been mathematically studied from several points of view [1], [2], [7], [4], [8], but has not been solved in an exactly manner. In this work, we formalize mathematically the idea of solution given in [1], we also give a physical interpretation in the case $L=0$ and obtain the quantum dynamics of a two-level atom interacting with an electromagnetic field [5]. This will be done through operators semigroup

Received: November 4, 2012

(c) 2013 Academic Publications, Ltd.

${ }^{\S}$ Correspondence author url: www.acadpubl.eu 
theory, characterizing the infinitesimal operator that generates the dynamics.

\section{Preliminaries}

In this section we give the definition of the Fock space, which is a new Hilbert space builded from a given Hilbert space.

Let $\mathcal{H}_{1}$ and $\mathcal{H}_{2}$ be Hilbert spaces; for each $\varphi_{1} \in \mathcal{H}_{1}$ and $\varphi_{2} \in \mathcal{H}_{2}$, the product $\varphi_{1} \otimes \varphi_{2}$ denotes the bilinear form that acts on $\mathcal{H}_{1} \times \mathcal{H}_{2}$ by the following rule:

$$
\left(\varphi_{1} \otimes \varphi_{2}\right)\left(\psi_{1}, \psi_{2}\right)=\left\langle\psi_{1}, \varphi_{1}\right\rangle_{1}\left\langle\psi_{2}, \varphi_{2}\right\rangle_{2},
$$

where $\langle\cdot, \cdot\rangle_{i}$ is the inner product in the Hilbert space $\mathcal{H}_{i}$.

Let $\mathcal{E}$ be the set of all finite linear combinations:

$$
\mathcal{E}=\left\{\sum_{i=1}^{n} \alpha_{i}\left(\varphi_{i_{1}} \otimes \varphi_{i_{2}}\right): \forall i=1, \ldots, n \quad \varphi_{i_{1}} \in \mathcal{H}_{1}, \varphi_{i_{2}} \in \mathcal{H}_{2}, \alpha_{i} \in \mathbb{C}\right\} .
$$

We define an inner product $\langle\cdot, \cdot\rangle$ over $\mathcal{E}$, as follows:

$$
\langle\varphi \otimes \psi, \eta \otimes \mu\rangle=\langle\varphi, \eta\rangle_{1}\langle\psi, \mu\rangle_{2} .
$$

This definition extends to all $\mathcal{E}$ by the bilinearity property and it can be proved that $\langle\cdot, \cdot\rangle$ is positive definite and well defined.

Definition 1. Let be the space $\mathcal{H}_{1} \otimes \mathcal{H}_{2}$ as the completation of $\mathcal{E}$ under the inner product $\langle\cdot, \cdot\rangle$ defined above. $\mathcal{H}_{1} \otimes \mathcal{H}_{2}$ will be called the tensor product of $\mathcal{H}_{1}$ and $\mathcal{H}_{2} ; \mathcal{H}_{1} \otimes \mathcal{H}_{2}$ is a Hilbert space.

We have the following two propositions whose proofs can be found in [6].

Proposition 1. If $\left\{\varphi_{k}\right\}$ and $\left\{\psi_{l}\right\}$ are orthonormal basis of $\mathcal{H}_{1}$ and $\mathcal{H}_{2}$ respectively, $\left\{\varphi_{k} \otimes \psi_{l}\right\}$ is an orthonormal basis of $\mathcal{H}_{1} \otimes \mathcal{H}_{2}$.

Proposition 2. Let $\left(M_{1}, \mu_{1}\right)$ and $\left(M_{2}, \mu_{2}\right)$ be measure spaces, and let consider the spaces $L^{2}\left(M_{1}, d \mu_{1}\right)$ and $L^{2}\left(M_{2}, d \mu_{2}\right)$. Then, there exists a unique isomorphism from $L^{2}\left(M_{1}, d \mu_{1}\right) \otimes L^{2}\left(M_{2}, d \mu_{2}\right)$ to $L^{2}\left(M_{1} \times M_{2}, d \mu_{1} \otimes d \mu_{2}\right)$ such that, $f \otimes g \mapsto f \cdot g$.

Definition 2. If $\left\{\mathcal{H}_{n}\right\}_{n=1}^{\infty}$ is a sequence of Hilbert spaces, let us define the direct sum space as the set

$$
\mathcal{H}=\bigoplus_{n=1}^{\infty} \mathcal{H}_{n}=\left\{\left\{x_{n}\right\}_{n=1}^{\infty}: x_{n} \in \mathcal{H}_{n}, \quad \sum_{n=1}^{\infty}\left\|x_{n}\right\|_{\mathcal{H}_{n}}^{2}<\infty\right\} .
$$


Again, the set $\mathcal{H}$ is a Hilbert space under the inner product $\langle\cdot, \cdot\rangle: \mathcal{H} \times \mathcal{H} \longrightarrow \mathbb{C}$ given by

$$
\left\langle\left\{x_{n}\right\}_{n=1}^{\infty},\left\{y_{n}\right\}_{n=1}^{\infty}\right\rangle_{\mathcal{H}}=\sum_{n=1}^{\infty}\left\langle x_{n}, y_{n}\right\rangle_{\mathcal{H}_{n}}
$$

Definition 3. Let $\mathcal{H}$ be a Hilbert space, we denote by $\mathcal{H}^{\otimes^{n}}$ the tensorial product of $n$-th order, i.e, $\mathcal{H}^{\otimes^{n}}=\mathcal{H} \otimes \mathcal{H} \otimes \cdots \otimes \mathcal{H}$. Let $\mathcal{H}^{0}=\mathbb{C}$, and we introduce

$$
\Gamma(\mathcal{H})=\bigoplus_{n=0}^{\infty} \mathcal{H}^{\otimes^{n}}
$$

$\Gamma(\mathcal{H})$ is called the Fock space over $\mathcal{H}$; this space will be separable if $\mathcal{H}$ is separable.

If $\mathcal{H}=L^{2}(\mathbb{R})$, then an element $\psi \in \Gamma\left(L^{2}(\mathbb{R})\right)$ is a sequence of functions that is of the form

$$
\psi=\left\{\psi_{0}, \psi_{1}, \psi_{2}, \cdots\right\}
$$

where $\psi_{0} \in \mathbb{C}$, and for all $k, \psi_{k} \in L^{2}\left(\mathbb{R}^{k}\right)$. From Definition 2 we have that the inner product and the norm in $\Gamma(\mathcal{H})$ are:

$$
\begin{aligned}
\langle\psi, \varphi\rangle_{\Gamma(\mathcal{H})} & =\overline{\psi_{0}} \varphi_{0}+\sum_{k=1}^{\infty}\left\langle\psi_{k}, \varphi_{k}\right\rangle_{L^{2}\left(\mathbb{R}^{k}\right)}, \\
\|\psi\|_{\Gamma(\mathcal{H})}^{2} & =\left|\psi_{0}\right|^{2}+\sum_{k=1}^{\infty}\left\langle\psi_{k}, \psi_{k}\right\rangle_{\mathcal{H}^{\otimes^{k}}} \\
& =\left|\psi_{0}\right|^{2}+\sum_{k=1}^{\infty} \int_{\mathbb{R}^{k}}\left|\psi_{k}\left(x_{1}, x_{2}, \cdots, x_{k}\right)\right|^{2} d x_{1} \cdots d x_{k}<\infty .
\end{aligned}
$$

To conclude this section we define for each $g \in \mathcal{H}$ the exponential vector $\psi(g)$, of argument $g$, whose components are given by:

$$
\psi_{0}=1, \quad \psi_{k}(g)=\frac{1}{\sqrt{k !}} g \otimes \cdots \otimes g
$$

it can be shown that the set of exponential vectors over the Hilbert space $\mathcal{H}$ is dense on $\Gamma(\mathcal{H})$. 


\section{Study of the QSDE}

Let us consider the following Quantum Stochastic Differential Equation (QSDE) [4], [8]:

$$
\left\{\begin{array}{l}
d V_{t}=\left[(W-I) d \Lambda_{t}-L^{*} W d A_{t}+L d A_{t}^{\dagger}-\left(i H+\frac{1}{2} L^{*} L\right) d t\right] V_{t} \\
V_{0}=I .
\end{array}\right.
$$

where $W, L$ and $H$ are the operators in the separable Hilbert space $\mathcal{H}$, while $d \Lambda_{t}, d A_{t}, d A_{t}^{\dagger}$ are the basic stochastic differentials in a symmetric Fock space.

According to the main results of quantum stochastic calculus [8], the solution of the previous QSDE, $\left\{V_{t}\right\}_{t \geq 0}$, is an adapted process of operators in $\Gamma^{s}\left(L^{2}\left(\mathbb{R}_{+}\right)\right) \otimes \mathcal{H}$, that describes the total evolution of the quantum system along with its environment. [2].

There is a one-parametric group $\left\{U_{t}\right\}_{t \in \mathbb{R}}$, canonically associated with $\left\{V_{t}\right\}_{t \geq 0}$;

In order to construct $U_{t}$ is necessary to consider the second quantization $\Theta_{t}$, over the symmetric Fock space

$$
\Gamma\left(L^{2}(\mathbb{R})\right)=\Gamma\left(L^{2}\left(\mathbb{R}_{-}\right)\right) \otimes \Gamma\left(L^{2}\left(\mathbb{R}_{+}\right)\right),
$$

of the unitary strongly continuous group of the translation operator $\theta_{t}$ on $L^{2}(\mathbb{R})$; which is defined for each $t \in \mathbb{R}$ by

$$
\begin{gathered}
\theta_{t} v(r)=v(r+t), \text { for all } v \in L^{2}(\mathbb{R}) \text { continuous, } \\
\Theta_{t} \psi(v)=\psi\left(\theta_{t} v\right), \text { for each } v \in L^{2}(\mathbb{R}),
\end{gathered}
$$

where $\psi(v)$ is the exponential vector associated with $v$.

The operator $\Theta_{t}$, can be identified with its extension to all space $\Gamma\left(L^{2}(\mathbb{R})\right) \otimes$ $\mathcal{H}$, and satisfies the cocycle property of $V_{t}$ with respect to $\Theta_{t}$ :

$$
V_{s+t}=\Theta_{s}^{*} V_{t} \Theta_{s} V_{s}, \forall s, t \geq 0 .
$$

The strong continuity of $V_{t}$ and $\Theta_{t}$ and the cocycle property of $V_{t}$, ensure the strong continuity of $U_{t}$.

We can define the unitary strong continuous one-parametric group $\left\{U_{t}\right\}_{t \in \mathbb{R}}$, by means of

$$
U_{t}= \begin{cases}\Theta_{t} V_{t} & t \geq 0 \\ V_{|t|}^{*} \Theta_{t} & t<0\end{cases}
$$


By the Stone theorem [9], $U_{t}$ and $\Theta_{t}$ are generated by some self adjoint operators (their Hamiltonians), i.e., for each $t \in \mathbb{R}$ we formally have,

$$
U_{t}=e^{-i t F}, \quad \Theta_{t}=e^{-i t E} .
$$

While the operator $E$ is known, the problem of completely characterize the operator $F$ is not easy since, in general $F$ is a singular operator and its domain is described by using boundary conditions.

\section{Infinitesimal generator of $\left\{U_{t}\right\}_{t \in \mathbb{R}}$}

The presents results were studied mathematically in [7]. There, it was considered the $L=0$ case, and $W$ was an unitary operator that commutes with the self adjoint and bounded operator $H$, that is the Hamiltonian of the system. The associated unitary group of the QSDE is defined for each $t \in \mathbb{R}$ by [1],

$$
U_{t} \Psi=\left(\psi_{0}, U_{t, 1} \psi_{1}, U_{t, 2} \psi_{2}, \ldots\right)
$$

where

$$
\Psi=\left(\psi_{0}, \psi_{1}, \psi_{2}, \ldots\right) \in \Gamma^{s}\left(L^{2}\left(\mathbb{R}_{*}\right)\right) \otimes \mathcal{H}
$$

with $\psi_{n}$ continuous for each $n \geq 1, \psi_{0} \in \mathbb{C}$ and $\mathbb{R}_{*}=\mathbb{R} \backslash\{0\}$. For each $n \geq 1$ we have,

$$
U_{t, n}= \begin{cases}U_{t, n} & t \geq 0 \\ U_{|t|, n}^{*} & t<0\end{cases}
$$

where

$$
\begin{aligned}
\left(U_{t, n} \psi_{n}\right)\left(x_{1}, \ldots, x_{n}\right)=e^{-i t H} \bigotimes_{j=1}^{n}\left\{I_{(-t, 0)}\left(x_{j}\right)\right. & v\left(x_{j}+t\right) W \\
& \left.+I_{\mathbb{R} \backslash(-t, 0)}\left(x_{j}\right) v\left(x_{j}+t\right)\right\} \otimes h,
\end{aligned}
$$

where $e^{-i t H}$ and $W$ can be identified with $I \otimes e^{-i t H}$ and $I \otimes W$, respectively. $I_{A}$ is the indicator function of the set $A$ and $\psi_{n}\left(x_{1}, \ldots, x_{n}\right)=v\left(x_{1}\right) \otimes \cdots \otimes v\left(x_{n}\right) \otimes h$, $h \in \mathcal{H}$. The definition of $U_{t, n}$ can be extended by density to all $L^{2}\left(\mathbb{R}_{*}^{n}\right) \otimes \mathcal{H}$ for each $n \in \mathbb{N}$.

In [1], an idea was given of how to characterize the corresponding infinitesimal generator; however, there was not given a complete proof of such a result. Here we will outline a characterization of the generator of the unitary group $U_{t}$. 
Let us consider the dense subspace of $\Gamma^{s}\left(L^{2}\left(\mathbb{R}_{*}\right)\right) \otimes \mathcal{H}$ given by

$$
\mathbb{F}=\left\{\Psi \in \Gamma^{s}\left(L^{2}\left(\mathbb{R}_{*}\right)\right) \otimes \mathcal{H}: \exists k \in \mathbb{N} \text { tal que } \psi_{n}=0 \forall n>k\right\}
$$

Let $F_{j}$ be the operator $F_{j}=\sum_{k=1}^{j} i \partial_{x_{k}}+H$, defined as

$$
D_{j}=\left\{\psi_{j} \in \mathcal{W}_{2,1}^{s}\left(\mathbb{R}_{*}^{j}\right) \otimes \mathcal{H}: \psi_{j}\left(0^{-}\right)=W \psi_{j}\left(0^{+}\right)\right\}
$$

where, $\mathcal{W}_{1,2}^{s}$ is the set of vectors on $\Gamma^{s}\left(L^{2}\left(\mathbb{R}_{*}\right)\right)$ whose $j$-th component is element of the Sobolev space $\mathcal{W}_{2,1}\left(\mathbb{R}_{*}^{j}\right) . \mathcal{W}_{2,1}^{s}\left(\mathbb{R}_{*}^{j}\right)$ is the projection of $\mathcal{W}_{2,1}^{s}$ on $j$-particle space $L^{2}\left(\mathbb{R}_{*}^{j}\right)$.

Theorem 1. The closure of the operator $F$ on $\Gamma^{s}\left(L^{2}\left(\mathbb{R}_{*}\right)\right) \otimes \mathcal{H}$ given by $F \Psi=\left(0, F_{1} \psi_{1}, \ldots, F_{n} \psi_{n}, \ldots\right)$ with domain $\mathcal{D}=\left\{\Psi \in \mathcal{F}: \psi_{j} \in D_{j}, \quad j \in\right.$ $\mathbb{N}\}$, is the infinitesimal generator of the strongly continuous group $\left\{U_{t}\right\}_{t \in \mathbb{R}}$ in $\Gamma^{s}\left(L^{2}\left(\mathbb{R}_{*}\right)\right) \otimes \mathcal{H}$.

In order to proof the previous theorem let us consider the one particle space, i.e., the first component of the Fock space.

Proposition 3. The closure of the operator $F_{1}=i \partial_{x}+H$ with domain $D_{1}=\left\{\psi \in \mathcal{W}_{2,1}\left(\mathbb{R}_{*}\right) \otimes \mathcal{H}: \psi\left(0^{-}\right)=W \psi\left(0^{+}\right)\right\}$is the infinitesimal generator of the strongly continuous group of unitary operators. $\left\{U_{t, 1}\right\}_{t \in \mathbb{R}}$ in $L^{2}\left(\mathbb{R}_{*}\right)$.

Proof. The proof is to show that $D_{1}$ is an essential domain for $F_{1}$. Because of a well known result for essential domains of infinitesimal generators [2]; is enough to proof the following:

1. $D_{1}$ is dense on $L^{2}\left(\mathbb{R}_{*}\right) \otimes \mathcal{H}$.

2. $D_{1}$ remains invariant under $U_{t, 1}$.

3. $D_{1} \subset D\left(F_{1}\right)$.

To illustrate the demonstration of these points, we will only consider the proof of point (3). Let $\psi \in D_{1}$, then

$$
\begin{aligned}
\| \frac{1}{t}\left(U_{t, 1}-I\right) \psi(x) & -\left(i \partial_{x}+H\right) \psi(x) \|^{2} \\
= & \int_{-\infty}^{-t}\left|\frac{1}{t}\left(U_{t, 1}-I\right) \psi(x)-\left(i \partial_{x}+H\right) \psi(x)\right|^{2} d x \\
& +\int_{-t}^{0}\left|\frac{1}{t}\left(U_{t, 1}-I\right) \psi(x)-\left(i \partial_{x}+H\right) \psi(x)\right|^{2} d x
\end{aligned}
$$




$$
+\int_{0}^{\infty}\left|\frac{1}{t}\left(U_{t, 1}-I\right) \psi(x)-\left(i \partial_{x}+H\right) \psi(x)\right|^{2} d x .
$$

The first and third integrals tends to zero as $t \rightarrow 0$, this is because of the fact that the operator $i F_{1}$ defined on $\mathcal{W}_{2,1}\left(\mathbb{R}_{*}\right) \otimes \mathcal{H}$ is the infinitesimal generator of the unitary group $\left(V_{t} \psi\right)(x)=e^{-i t H} \psi(x+t)$, for each continuous function $\psi \in L^{2}\left(\mathbb{R}_{*}\right) \otimes \mathcal{H}$.

To perform the the second integral, we can introduce the function

$$
\tilde{\psi}(x)= \begin{cases}W \psi(x) & , x \geq 0 \\ \psi(x) & , x<0\end{cases}
$$

by substituting in the second integral we obtain

$$
\begin{aligned}
\int_{-t}^{0} \mid \frac{1}{t}\left(U_{t, 1}-I\right) \psi(x) & -\left.\left(i \partial_{x}+H\right) \psi(x)\right|^{2} d x \\
& =\int_{-t}^{0}\left|\frac{1}{t}\left(e^{-i t H} W \psi(x+t)-\psi(x)\right)-\left(i \partial_{x}+H\right) \psi(x)\right|^{2} d x \\
& =\int_{-t}^{0}\left|\frac{1}{t}\left(e^{-i t H} \tilde{\psi}(x+t)-\tilde{\psi}(x)\right)-\left(i \partial_{x}+H\right) \tilde{\psi}(x)\right|^{2} d x \rightarrow 0,
\end{aligned}
$$

when $t \rightarrow 0$, the last limit holds because the operator $-\partial_{x}+i H$ defined in $\mathcal{W}_{2,1}\left(\mathbb{R}_{*}\right) \otimes \mathcal{H}$ is the infinitesimal generator of the unitary group

$$
\left(V_{t} \psi\right)(x)=e^{-i t H} \psi(x+t), \quad \psi \in L^{2}\left(\mathbb{R}_{*}\right) \otimes \mathcal{H} .
$$

In this way we have proved that $D_{1} \subset D\left(F_{1}\right)$.

The proof of Theorem 1 can be completed by taking the second quantization of the unitary group and its generator in proposition 3. This method allows to determine the essential domain of the operator $F$.

\section{Physical Applications}

\subsection{Linear Motion of a Photon with Local Interaction}

If we consider the group $U_{t, 1}$ in $L^{2}\left(\mathbb{R}_{*}\right)$, i.e., if $\mathcal{H}=\mathbb{C}$; and we take the operators $W=e^{i \theta}$ and $H=0$ with $\theta$ a real nonzero, then we obtain the strongly continuous group of unitary operators

$$
U_{t, 1}^{\theta}= \begin{cases}U_{t, 1}^{\theta+} & , t \geq 0 \\ U_{|t|, 1}^{\theta *} & , t<0\end{cases}
$$


where, for each $t \geq 0$

$$
\left(U_{t, 1}^{\theta+} \psi\right)(x)=\psi(x+t)\left\{e^{i \theta} I_{(-t, 0)}(x)+I_{\mathbb{R} \backslash(-t, 0)}(x)\right\} .
$$

Physically, this system models the evolution of a photon that moves across an optical axis (the real line), from right to left and, permanently interacts at the origin, with an external quantum system, which imprints to it a phase shift equal to $e^{i \theta}$.

According to proposition 3, the infinitesimal generator of $U_{t, 1}^{\theta}$ is the closure of the operator $F_{1}^{\theta}=i \partial_{x}$ with domain $D_{1}=\left\{\psi \in \mathcal{W}_{2,1}\left(\mathbb{R}_{*}\right): \psi\left(0^{-}\right)=\right.$ $\left.e^{i \theta} \psi\left(0^{+}\right)\right\}$.

If for each $t \in \mathbb{R}$ we define on $L^{2}\left(\mathbb{R}_{*}\right)$ the family of operators given by

$$
U_{t, 1}^{\theta, \alpha}= \begin{cases}U_{t, 1}^{\theta, \alpha+} & , t \geq 0 \\ U_{|t|, \alpha *}^{\theta *} & , t<0\end{cases}
$$

where, for each $t \geq 0$

$$
\left(U_{t, 1}^{\theta, \alpha+} \psi\right)(x)=\psi(x+t) e^{i \theta \int_{0}^{t} V_{\alpha}(x+t-\tau) d \tau}
$$

with $V_{\alpha}=(2 \pi \alpha)^{-\frac{1}{2}} e^{-\frac{x^{2}}{2 \alpha}}$, then the following propositions can be established:

Proposition 4. The family $\left\{U_{t, 1}^{\theta, \alpha}\right\}_{t \in \mathbb{R}}$ is a strongly continuous group of unitary operators in $L^{2}\left(\mathbb{R}_{*}\right)$ whose infinitesimal generator is the operator $F_{1}^{\theta, \alpha}=\partial_{x}+i \theta V_{\alpha}$ defined on $\mathcal{W}_{2,1}\left(\mathbb{R}_{*}\right)$.

Proposition 5. $U_{t, 1}^{\theta, \alpha} \rightarrow U_{t, 1}^{\theta}$ when $\alpha \rightarrow 0^{+}$strongly in $\mathcal{B}\left(L^{2}\left(\mathbb{R}_{*}\right)\right)$. Then, $F_{1}^{\theta, \alpha} \rightarrow F_{1}^{\theta}$ when $\alpha \rightarrow 0^{+}$strongly in the resolvers sense.

According to these propositions, the dynamics generated by the operator $F_{1}^{\theta}$, is the limit of the dynamics generated by the operator $F_{1}^{\theta, \alpha}$, when $\alpha \rightarrow 0^{+}$.

\subsection{Two-Level Atom Model}

In quantum optics the basic model consists of some physical system of interest, for example, a two-level atom, a cloud of atoms, or an atom in a cavity, in interaction with an electromagnetic field. The interaction between the electromagnetic field and the system is described by quantum electrodynamics [3].

Suppose we are studying a two-level atom in interaction with the electromagnetic field; in this case the operarots $W, L$ y $H$ acting over $\mathcal{H}=\mathbb{C}^{2}$, are 
given by

$$
W=I, \quad L=\gamma \sigma_{-}=\left(\begin{array}{ll}
0 & 0 \\
\gamma & 0
\end{array}\right), \quad H=\frac{\hbar \omega_{0}}{2} \sigma_{z}=\left(\begin{array}{cc}
\frac{\hbar \omega_{0}}{2} & 0 \\
0 & -\frac{\hbar \omega_{0}}{2}
\end{array}\right),
$$

where $\hbar$ is the Planck constant, $\gamma \geq 0$ is a decay parameter, $\omega_{0}$ the atomic frecuency and $\hbar \omega_{0}$ the diference of energy between the two levels. The interaction with the electromagnetic field is through the QSDE:

$$
\left\{\begin{array}{l}
d V_{t}=\left[L d A_{t}^{\dagger}-L^{*} d A_{t}-\left(i H+\frac{1}{2} L^{*} L\right) d t\right] V_{t} \\
V_{0}=I .
\end{array}\right.
$$

Now, taking into account the two-level atom model, we have

$$
\left\{\begin{array}{l}
d V_{t}=\left[\gamma \sigma_{-} d A_{t}^{\dagger}-\gamma \sigma_{+} d A_{t}-\frac{\gamma^{2}}{2} \sigma_{+} \sigma_{-} d t-i \frac{\hbar}{2} \sigma_{z}\right] V_{t} \\
V_{0}=I
\end{array}\right.
$$

whose formal solution is:

$$
V_{t}=\mathcal{T} \exp \left\{\int_{0}^{t} \gamma \sigma_{-} d A_{s}^{\dagger}-\gamma \sigma_{+} d A_{s}-i \frac{\hbar \omega_{0}}{2} \sigma_{z} d s\right\}
$$

where $\mathcal{T}$ denotes the time-ordered product of operators.

\section{Summary}

By a different approach from that developed by A. Chebotarev [1] and [2], we studied the problem of characterizing the solution of a QSDE in the framework of the theory of operators. The method outlined in third section allows to characterize the infinitesimal generator of the unitary group $\left\{U_{t}\right\}_{t \in \mathbb{R}}$. This unitary group admits the physical interpretation described in the fourth section and it is the strong limit of a family of translations perturbed by a Gaussian potential. Formally, the family of infinitesimal generators of these unitary groups is the singular operator $\partial_{x}+\delta_{0}$ with $\delta_{0}$ the Dirac delta measure concentrated at the origin; in our approach this singular operator was rigorously defined as the operator $F_{1}^{\theta}$.

In physics, there also exist some systems that could be modeled through equations like (3.1), such is the case of the equation for the amplitude of a laser, which must consider noise effects due to both the quantum nature of the laser, and its interaction with the environment [10]. Such noise could be modeled through stochastic processes and modeled in a QSDE as (3.1); however, to complete the connection further studies are still required. 


\section{References}

[1] A.M. Chebotarev, Lectures on Quantum Probability; SMM Aportaciones Matemáticas (Textos nivel avanzado), 14, México, 2000.

[2] A.M. Chebotarev, What is a quantum stochastic differential equation from the point of view of functional analysis?, Math. Notes, 71 (2002), 408-427.

[3] C. Cohen Tannoudji, J. Dupont Roc, G. Grynberg, Photons and Atoms: Int. to Quantum Electrodynamics, Wiley (1989).

[4] Alexander S. Holevo, Statistical Structure of Quantum Theory, SpringerVerlag, New York (2001).

[5] Luc Bouten, Applications of Quantum Stochastic Processes in Quantum Optics, Chapter in: Quantum potential theory. Lecture Notes in Mathematics (1954), Springer, Berlin, 277-307, 2008.

[6] M. Reed, W. Barry Simon, Methods of Modern Mathematical Physics, I, Academic Press, New York (1970).

[7] O. González-Gaxiola, R. Quezada, On the infinitesimal generator of a quantum stochastic differential equation, Stochastic Models., 22 (2006), 561-572.

[8] K.R. Parthasarathy, An Introduction to Quantum Stochastic Calculus, Birkhäuser, Basel (1992).

[9] J. Weidmann, Linear Operators in Hilbert Spaces, Graduate Texts in Mathematics, 68, Springer-Verlag, New York (1980).

[10] H. Risken, The Fokker-Planck Equation: Methods of Solution and Applications, Springer-Verlag (1989). 